Int. J. Morphol.,

34(3):836-841, 2016

\title{
Vitrificación de Ovocitos Bovinos Parcialmente Madurados de la Raza Frisón Rojo Chileno
}

\author{
Vitrification of Partially Matured Bovine Oocytes of the Chilean Red Friesian Breed
}

\author{
M. Silva ${ }^{*, * *} \&$ N. Calderón*
}

SILVA, M. \& CALDERÓN, N. Vitrificación de ovocitos bovinos parcialmente madurados de la raza Frisón Rojo Chileno. Int. J. Morphol., 34(3):836-841, 2016.

RESUMEN: Se evaluó la tasa de maduración in vitro post descongelación de ovocitos bovinos, de la raza Frisón Rojo Chileno, parcialmente madurados y vitrificados. Complejos Cúmulus-ovocito fueron obtenidos por aspiración folicular, clasificados morfológicamente y aleatoriamente cultivados in vitro en TCM199 (10\% Suero Fetal Bovino (SFB), $50 \mathrm{mg} / \mathrm{mL}$ gentamicina, 0,2 mM piruvato de sodio, $0,08 \mu \mathrm{g} / \mathrm{mL}$ FSH, $1 \mu \mathrm{g} / \mathrm{mL} \mathrm{LH} \mathrm{y} 1 \mu \mathrm{g} / \mathrm{mL}$ estradiol) en los grupos: a) control ( $\mathrm{n}=137)$, madurados por $24 \mathrm{~h}$ a $38,5^{\circ} \mathrm{C}$, $5 \% \mathrm{CO}_{2}$ y $99 \%$ humedad y, b) tratamiento $(\mathrm{n}=156)$, madurados por $6 \mathrm{~h}$, parcialmente denudados, e incubados hasta completar $20 \mathrm{~h}$, para luego ser vitrificados por el método Open Pulled Straws (OPS). Los ovocitos fueron expuestos a la solución de vitrificación uno (SV1) (Buffer Fosfato Salino (PBS), $10 \%$ Etilenglicol (EG), 10\% DMSO) por 30 s, posteriormente traspasados a la SV2 (PBS, $20 \%$ EG, $20 \%$ DMSO) por $25 \mathrm{~s}$. Inmediatamente los ovocitos fueron cargados en pajuelas francesas estiradas (OPS) y sumergidos en nitrógeno líquido. Las ovocitos fueron descongelados introduciéndolos en una secuencia de soluciones con concentraciones decrecientes de sucrosa $(0,3$; 0,15 y 0 M respectivamente). Finalmente, los ovocitos continuaron con la maduración por 4 h adicionales. Posterior al periodo de maduración, los ovocitos de ambos grupos fueron fijados, teñidos y evaluados. Las proporciones de ovocitos en Metafase I (MI), Metafase II (MII) y degenerados fueron comparadas mediante el test de Chi cuadrado. La vitrificación aumentó ( $\mathrm{p} \leq 0,05)$ el porcentaje de pérdida y de ovocitos dañados en comparación al control. Además, aumentó $(\mathrm{p} \leq 0,05)$ la tasa de ovocitos en MI y el número de ovocitos degenerados, y redujo el porcentaje de ovocitos MII, en comparación al control. Por tanto, la vitrificación por el método Open Pulled Straw de ovocitos parcialmente madurados in vitro es una alternativa viable para la conservación de material genético de hembras Frisón Rojo Chileno.

PALABRAS CLAVE: Ovocitos; Vitrificación; Maduración in vitro; Frisón Rojo Chileno.

\section{INTRODUCCION}

Uno de los mayores desafíos aun existentes para la criobiología reproductiva en mamíferos, y específicamente en bovinos, es la criopreservación de la célula germinal femenina, el ovocito, lo cual permitiría la conservación de material genético materno para distintos fines (de Souza et al., 2011). Las principales dificultades enfrentadas para lograr criopreservar este tipo celular tienen relación con su gran volumen, la presencia de la zona pelúcida, su alto contenido de lípidos, presencia de gránulos corticales, y el hecho de que para que pueda continuar y concluir el proceso de división meiótica, el sincitio conformado por las células del cumulus oophorus y el ovocito debe mantenerse intacto, ósea que las uniones gap que interconectan está red celular no deben ser afectadas por el proceso de criopreservación (Hyttel et al., 2000; Saragusty \& Arav, 2011). Por otra parte se ha demostrado que el estado de maduración nuclear del ovocito afecta su sensibilidad a la criopreservación, lo cual ha sido reportado en diversas especies de animales domésticos, como equinos (Tharasanit et al., 2006), búfalos (Sharma \& Loganathasamy, 2007) y bovinos (Hochi et al., 1998; Men et al., 20002).

Debido a lo anterior, los ovocitos que generalmente son criopreservados son aquellos que han sido sometidos a un proceso de maduración in vitro (Albarracín et al., 2005; Morató et al., 2008; Zhou et al., 2010;) y se encuentran en estado de Metafase II (MII). En estos, el control del reinicio de la meiosis efectuado por las células del cúmulus oophorus y de la corona radiata, ya se ha desarrollado y por tanto su permanencia e integridad ya no es requerida. Sin embargo,

\footnotetext{
* Escuela de Medicina Veterinaria, Facultad de Recursos Naturales, Universidad Católica de Temuco, Temuco, Chile.

** Núcleo de Investigación en Producción Alimentaria, Universidad Católica de Temuco, Temuco, Chile.
} 
ovocitos maduros presentan una mayor sensibilidad a sufrir daños a nivel nuclear producto de la congelación, debido a la dispersión de los cromosomas por la ruptura de los microtúbulos del huso meiótico que se ha conformado durante la reanudación de la meiosis (Aman \& Parks, 1994; Vieira et al., 2002; Albarracín et al., 2005). Ya que los ovocitos inmaduros, en estado de Vesícula Germinal, no presentan un huso meiótico organizado y por tanto una potencial menor sensibilidad nuclear a sufrir daños por el proceso de congelación, se ha intentado también la criopreservación de ovocitos en este estado (Otoi et al., 1995; Vieira et al., 2002; Dhali et al., 2000; Sharma \& Loganathasamy; Zhou et al.). Sin embargo, como ya fuese mencionado el potencial daño a nivel de las uniones gap del sincitio conformado por las células del cúmulus oophorus, que posteriormente dificultará la maduración, tanto nuclear como citoplasmática de los ovocitos, y su posterior potencial de desarrollo, es una limitante.

Dado lo anterior, una tercera alternativa que ha sido evaluada es la criopreservación de ovocitos bovinos parcialmente madurados, posterior a 18-22 h de cultivo in vitro (Vatja et al., 1998; Hyttel et al.; Hou et al., 2005; Sharma \& Loganathasamy) la cual ha demostrado incrementar la tasa de éxito de la criopreservación al generar menores efectos sobre el potencial de desarrollo posterior de los ovocitos una vez fecundados (Vatja et al., 1998; Hyttel et al.). Sin embargo, esta alternativa ha sido aún poco explorada y los efectos de la criopreservación sobre la maduración nuclear del ovocito escasamente estudiados.

El desarrollo de la vitrificación, una técnica que genera la rápida solidificación de una solución, mediante la aplicación a altas tasas de congelación y altas concentraciones de crioprotectores, sin la formación de cristales de hielo (Rall \& Fahy, 1985); permitió mejorar las tasas de sobrevivencia de ovocitos en comparación a la congelación tradicional (Vatja et al., 1998). Adicionalmente, el desarrollo de nuevas técnicas y dispositivos relacionados a la vitrificación, como Solid Surface Vitrification, Cryoloop, Open Pulled Straw entre otras (Saragusty \& Arav) que logran incrementar aún más la tasa de congelación y descongelación, hasta en 10 veces (Vajta et al.), han generado nuevas alternativas para la congelación de embriones y ovocitos, con potenciales mayores tasas de éxito que las obtenidas con la vitrificación tradicional (Vajta et al., 1997a; Vatja et al., 1998; Hyttel et al.). Estudios de la morfología, biología y criopreservación de ovocitos en distintas especies, y en particular en distintas razas bovinas, han demostrado el valor de esta técnica en la conservación y multiplicación de genotipos locales valiosos o en peligro (Li et al., 2002; de Souza et al.).
El presente estudio tiene como objetivo evaluar las características morfológicas, tanto citoplasmáticas como nucleares y la tasa de maduración in vitro post descongelación de ovocitos bovinos, de la raza Frisón Rojo Chileno, parcialmente madurados in vitro y vitrificados por el método Open Pulled Straw (OPS).

\section{MATERIAL Y MÉTODO}

\section{Colección de los Complejos Cumulus-Ovocito (CCOs) y} maduración in vitro. Ovarios de vacas Frisón Rojo Chileno, fueron colectados en el frigorífico local y transportados al laboratorio dentro de una hora posterior al sacrificio de los animales. Se aspiraron folículos entre 2 y $8 \mathrm{~mm}$ de diámetro y los CCOs se clasificaron morfológicamente en base a las características de su citoplasma y cumulus oophorus. Grupos de 10-13 CCOs fueron depositados en gotas de 50 $\mathrm{mL}$ de medio TCM199 (10\% Suero Fetal Bovino (SFB), 50 $\mathrm{mg} / \mathrm{mL}$ gentamicina, $0,2 \mathrm{mM}$ piruvato de sodio, $0,08 \mu \mathrm{g} /$ $\mathrm{mLl} \mathrm{FSH,} 1 \mathrm{ug} / \mathrm{mL}$ LH y $1 \mu \mathrm{g} / \mathrm{mL}$ estradiol), cubiertas por aceite mineral, y aleatoriamente distribuidos en los siguientes grupos: a) Control (maduración tradicional; $n=137$ ): incubación por $24 \mathrm{~h}$ a $38,5^{\circ} \mathrm{C}, 5 \% \mathrm{CO}_{2}$ y $99 \%$ humedad relativa, y b) Tratamiento (maduración parcial; $\mathrm{n}=156$ ) incubación por 6 horas, bajo las mismas condiciones descritas para el grupo control, tras lo cual se realizó la denudación parcial de las células del cúmulus oophorus mediante pipeteo reiterado suave, en medio Buffer Fosfato Salino (PBS) suplementado con $5 \%$ SFB, dejando 2-3 capas de células adheridas al ovocito. Posteriormente fueron lavados en medio de maduración a $38,5^{\circ} \mathrm{C}$ para ser devueltos a sus respectivas gotas de maduración hasta completar 20 h de incubación, momento en el cual fueron vitrificados.

Vitrificación de ovocitos parcialmente madurados (grupo tratamiento). Para este procedimiento se utilizó el protocolo descrito por Vatja et al. (1998). En resumen, pajuelas francesas $(0,25 \mathrm{~mL}$; Minitube, Germany) fueron calentadas en su tercio medio sobre una platina temperada, luego mediante tracción manual de ambos extremos se estiraron reduciendo el diámetro en un $50 \%$. Posteriormente se cortaron en la porción más delgada, obteniendo 2 pajuelas estiradas. Todos los procedimientos descritos a continuación fueron realizados en medios temperados sobre una platina $\left(41^{\circ} \mathrm{C}\right)$ y a una temperatura ambiente de $25^{\circ} \mathrm{C}$. Grupos de $4-5$ ovocitos fueron lavados en solución de equilibrio (PBS, $5 \%$ SFB) y mantenidos en la misma solución hasta depositarlos en la solución de vitrificación uno (SV1; 10 \% Etilenglicol, 10 \% DMSO disuelto en solución de equilibrio) por un periodo de $30 \mathrm{~s}$, posteriormente fueron traspasados a la SV2 (20\% EG, $20 \%$ DMSO y Sucrosa 0,5 M disuelto en solución de equilibrio) 
por 25 s. Desde esta solución, los ovocitos fueron cargados por capilaridad en las pajuelas estiradas en una columna de aproximadamente $3 \mu \mathrm{L}$ de SV2, para luego sumergirlas directamente en nitrógeno líquido, siendo almacenadas en este medio al menos por 5 días.

Para la descongelación las pajuelas fueron retiradas individualmente desde el nitrógeno líquido y el extremo delgado, conteniendo los ovocitos, fue rápidamente sumergido en la solución de descongelación uno (SD1; 0,25 M de Sucrosa en solución de equilibrio), en la cual los ovocitos fueron liberados al descongelarse la columna de SV2 que los contenía. Luego de permanecer $5 \mathrm{~min}$ en la SD1 los ovocitos fueron transferidos a SD2 (0,15 M Sucrosa en solución de equilibrio) por otros $5 \mathrm{~min}$. Posteriormente, fueron lavados 2 veces por 5 min cada una en solución de equilibrio y una vez en medio de maduración, para luego ser transferidos a microgotas $(50 \mathrm{~mL})$ de medio de maduración, para continuar con el proceso de maduración in vitro por $4 \mathrm{~h}$ adicionales.

Evaluación morfología nuclear y citoplasmática de ovocitos. Una vez completado el periodo de maduración in vitro, tanto para el grupo control como tratamiento, y en forma independiente, los ovocitos fueron denudados de las células del cúmulus oophorus aun adheridas mediante agitación suave (vortex) por $2 \mathrm{~min}$. Luego, los ovocitos fueron montados en portaobjetos en grupos de 15-20, fijados en alcohol acético por $12 \mathrm{~h}$ y teñidos con aceto-orceina $1 \%$ por $15 \mathrm{~min}$. Se evaluaron los porcentajes de ovocitos perdidos y dañados, y las características morfológicas nucleares, de acuerdo a la siguiente clasificación: a) Metafase I (MI): ovocitos en los que no se evidenció la membrana nuclear, con un ordenamiento de los cromosomas formando una placa metafásica en el plano ecuatorial del huso meiótico. En esta categoría también fueron incluidos aquellos ovocitos que habiendo reasumido la división meiótica no alcanzaron el estado de Metafase II, y b) Metafase II (MII): ovocitos en los cuales se observó la extrusión del primer corpúsculo polar y los cromosomas se observan ordenados formando una placa en el plano ecuatorial del huso meiótico. Además se evaluaron las características morfológicas citoplasmáticas clasificando como ovocitos degenerados aquellos que presentaban deformaciones y/o vacuolas a nivel citoplasmático. Además, fueron considerados en esta categoría aquellos ovocitos en los cuales no fue posible identificar ninguna estructura nuclear.

El porcentaje de pérdida y daño, de ovocitos en estado MI, MII y degenerados fue comparado entre grupos mediante la prueba de Chi cuadrado. El nivel de significancia establecido fue de $5 \%$.

\section{RESULTADOS}

Un total de 156 y 137 CCOs fueron madurados in vitro en 7 réplicas, para el grupo tratamiento y control respectivamente. El proceso de vitrificación/descongelación aumentó en forma significativa ( $\mathrm{p} \leq 0,05)$ el porcentaje de pérdida y de ovocitos dañados en comparación al grupo control. Los factores que determinan el porcentaje de pérdida/ daño se detallan en la Tabla I. Por otra parte, la vitrificación/

Tabla I. Factores asociados a la pérdida y daño de ovocitos bovinos durante el proceso de maduración in vitro asociado (grupo tratamiento) o no (grupo control) a la vitrificación mediante la técnica Open Pulled Straw (OPS).

\begin{tabular}{lcc}
\hline & \multicolumn{2}{c}{ Pérdida y daño de ovocitos (n; \%) } \\
\cline { 2 - 3 } & $\begin{array}{c}\text { Tratamiento } \\
(\mathbf{n}=\mathbf{1 5 6})\end{array}$ & $\begin{array}{c}\text { Control } \\
(\mathbf{n}=\mathbf{1 3 7})\end{array}$ \\
\hline Ovocitos perdidos & $5(3,2)^{\mathrm{a}}$ & $0(0)^{\mathrm{b}}$ \\
Ovocitos dañados & $25(16)^{\mathrm{a}}$ & $5(3,7)^{\mathrm{b}}$ \\
Ovocitos dañados por FZP* & $20(80)^{\mathrm{a}}$ & $0(0)^{\mathrm{b}}$ \\
Ovocitos desintegra dos* & $5(20)^{\mathrm{a}}$ & $5(100)^{\mathrm{b}}$ \\
\hline
\end{tabular}

a,b: Valores con superíndices distintos dentro de la misma fila difieren estadísticamente ( $\mathrm{p} \leq 0,05)$.

*: porcentaje calculado sobre el total de ovocitos dañados. FZP: Fractura de zona pelúcida.

Tabla II. Características nucleares y citoplasmáticas de ovocitos bovinos luego del proceso de maduración in vitro asociado (grupo tratamiento) o no (grupo control) a la vitrificación mediante la técnica Open Pulled Straw (OPS).

\begin{tabular}{lcccc}
\hline & $\begin{array}{c}\text { n ovocitos } \\
\text { evaluados }\end{array}$ & Ovocitos MI & Ovocitos MII & Ovocitos degenerados \\
\cline { 2 - 5 } Control & 132 & $\mathbf{n}(\%)$ & $\mathbf{n ~ ( \% )}$ & $\mathbf{n}(\%)$ \\
Tratamiento & 126 & $50(12,1)^{\mathrm{a}}$ & $98(74,2)^{\mathrm{a}}$ & $18(13,6)^{\mathrm{a}}$ \\
\hline
\end{tabular}

a,b: Valores con superíndices distintos dentro de la misma columna difieren estadísticamente $(\mathrm{p} \leq 0,05)$. 
descongelación aumentó también $(\mathrm{p} \leq 0,05)$ la tasa de ovocitos en MI y el número de ovocitos degenerados en comparación al grupo control (Tabla II). Contrariamente, la tasa de maduración in vitro (ovocitos en MII) fue negativamente $(\mathrm{p} \leq 0,05)$ afectada por el proceso de vitrificación/descongelación (Tabla II).

\section{DISCUSION}

Los resultados de este estudio confirman que a pesar de la reducción en la tasa de maduración nuclear en comparación a los ovocitos control, la vitrificación de ovocitos parcialmente madurados es una alternativa efectiva para la conservación de material genético proveniente de hembras bovinas de la raza Frisón Rojo Chileno. Aunque ha sido sugerido que el mejor indicador para evaluar la efectividad de la criopreservación de ovocitos es la generación de descendencia viable (Hamano et al., 1992), estudios como el presente u otros similares (Martins et al., 2005; Sharma \& Loganathasamy) permiten generar información relevante, al evaluar el efecto de la criopreservación sobre las características nucleares y citoplasmáticas de los ovocitos, y se constituyen en pasos previos a la realización de estudios de producción in vitro de embriones a partir de ovocitos criopreservados.

El procedimiento de vitrificación/descongelación mediante OPS incrementó significativamente el porcentaje de pérdida de ovocitos; sin embargo, este porcentaje fue bajo $(3,2 \%)$ comparativamente al $11 \%$ reportado por nuestro grupo de trabajo, al vitrificar embriones bovinos por el método OPS (Silva \& Berland, 2004), o por otros grupos al vitrificar ovocitos bovinos con la misma técnica $(16,7 \%$; Albarracín et al.). Por otra parte, la pérdida de ovocitos registrada en nuestro estudio fue menor a la reportada al vitrificar ovocitos bovinos por el método tradicional (15\%; Dhali et al. y 21,9\%; Sharma \& Loganathasamy) o por la técnica Solid Surface Vitrification (18,5\%; Dinnyés et al., 2000). Adicionalmente, el porcentaje de ovocitos dañados (16\%) fue también significativamente mayor en el grupo tratamiento en comparación al grupo control. Proporcionalmente la variable que contribuyó mayoritariamente (80\%) al daño de los ovocitos vitrificados/descongelados fue la fractura de zona pelúcida (ZP), la cual se genera por un cambio no uniforme en la solución de congelación durante el paso del estado líquido a sólido (Rall \& Fahy). Porcentajes comparables $(77 \%)$ han sido reportados para ovocitos de búfalo (Dhali et al.) y ovocitos bovinos (42,3\%; Sharma \& Loganathasamy) vitrificados utilizando el método tradicional (pajuelas francesas convencionales). Sin embargo, cuando se evalúa el porcentaje de fractura de ZP sobre el total de embriones u ovocitos vitrificados, los valores reportados para la técnica OPS son bajos y varían entre un 4,8 \% (Silva \& Berland), $1 \%$ (Vatja et al., 1997b) y un $0 \%$ (Vatja et al., 1998). Este menor daño se debería a que a pesar que el OPS permite lograr mayores tasas de congelación y descongelación, las que alcanzan los $20,000^{\circ} \mathrm{C} / \mathrm{min}$ (Vatja et al., 1998), con este método no ocurren cambios de presión en la solución de congelación durante el proceso, los que sí se observan al utilizar pajuelas cerradas (congelación y vitrificación tradicional), en donde los porcentajes de fractura de ZP descritos alcanzan el 20-27 \% (Vatja et al., 1997b).

Actualmente las mayores tasas de desarrollo de blastocistos se han logrado al vitrificar ovocitos MII (Martino et al., 1996; Men et al.), siendo estas menores en el caso de ovocitos inmaduros (Prentice-Biensch et al., 2012; Vieira et al.), lo que sugiere que la tolerancia de los ovocitos aumenta con el estado de maduración de los mismos. Tasas de desarrollo de blastocistos de un $25 \%$, en comparación a ovocitos no criopreservados (48\%), obtenidas utilizando el mismo procedimiento de vitrificación de este estudio, son un indicador de que la vitrificación de ovocitos en estadíos previos a completar su maduración, es una alternativa altamente efectiva para la conservación de ovocitos (Vatja et al., 1998).

Indicios de la factibilidad de criopreservar ovocitos parcialmente madurados fueron sugeridos ya en estudios pioneros en congelación de ovocitos (Mapes \& Wells, 1977), en donde se intentó congelar este tipo celular luego de un periodo de maduración in vitro de $12 \mathrm{~h}$. Posteriormente, tasas de desarrollo de blastocistos de $1 \%$ se han obtenido con la vitrificación de ovocitos madurados por $8 \mathrm{~h}$, que se encuentran en estadio de disolución de la Vesícula Germinal (Men et al.). Estudios más recientes han demostrado que ovocitos madurados por 18-22 $\mathrm{h}$ ya han terminado su primera división meiótica y se encuentran mayoritariamente en Telofase I (Gordon, 2003; Sharma \& Loganathasamy), estado en el cual los microtúbulos no están ordenados en un huso, disminuyendo de esta manera la sensibilidad nuclear a la congelación del ovocito; por otra parte, el control ejercido sobre el reinicio de la división meiótica por las células del cumulus oophorus ya fue efectuado, minimizando de esta manera los riesgos existentes en la congelación de ovocitos en estado MII y vesícula germinal respectivamente.

El presente estudio aporta información relevante en cuanto al estado de la maduración nuclear de ovocitos bovinos madurados parcialmente y vitrificados por el método OPS, que se condice con tasas de desarrollo embrionario obtenidas en ovocitos criopreservados utilizando el mismo protocolo (Vatja et al., 1998; Hyttel et al.). 
Podemos concluir entonces que la congelación de ovocitos antes que completen el periodo de maduración in vitro de 24-26 h (Sirad et al., 1989; Hochi et al.; Smiljakcovic \& Tomek, 2006) requerido para alcanzar el estado de MII, ha demostrado ser una alternativa viable para la conservación de material genético de hembras bovinas de la raza Frisón Rojo Chileno. Estudios complementarios para evaluar la tasa de segmentación, de producción de blastocistos y eventualmente de crías viables nacidas son necesarios para determinar el potencial de desarrollo de los ovocitos criopreservados.

SILVA, M. \& CALDERÓN, N. Vitrification of partially matured bovine oocytes of the Chilean Red Friesian breed. Int. J. Morphol., 34(3):836-841, 2016.

SUMMARY: Post thawing in vitro maturation rate was evaluated for partially matured vitrified oocytes from Chilean Red Friesian cattle. Cumulus-Oocytes Complexes were obtained by follicular aspiration, classified by morphology and randomly in vitro matured in TCM199 (10\% Bovine Fetal Serum (BFS), $50 \mathrm{mg} / \mathrm{mL}$ gentamicine, $0.2 \mathrm{mM}$ sodium piruvate, $0.08 \mu \mathrm{g} / \mathrm{ml} \mathrm{FSH,} 1 \mu \mathrm{g} / \mathrm{mL} \mathrm{LH}$ and $1 \mu \mathrm{g} / \mathrm{mL}$ estradiol) in the following groups: a) control ( $\mathrm{n}=137)$, matured for $24 \mathrm{~h}$ at $38.5{ }^{\circ} \mathrm{C}, 5 \% \mathrm{CO}_{2}$ y $99 \%$ humidity, and b) treatment $(n=156)$, matured for $6 \mathrm{~h}$, partially denuded, and incubated until completion of $20 \mathrm{~h}$. Then, oocytes were vitrified by the Open Pulled Straws (OPS) method. Oocytes were exposed to vitrification solution one (VS1) (Phosphate Buffered Saline (PBS), $10 \%$ Ethylen glycol (EG), $10 \%$ DMSO) for $30 \mathrm{~s}$, then they were exposed to VS2 (PBS, 20\% EG, 20\% DMSO) for 25 s. Afterwards, oocytes were loaded into open pulled straws and submerged into liquid nitrogen. Oocytes were thawed by exposure to sequential solutions with decreasing concentrations of sucrose $(0.3 ; 0.15$ y $0 \mathrm{M}$ respectively). Finally, oocytes continued the in vitro maturation for 4 additional hours. After completion of maturation period oocytes from both groups were fixated, stained and evaluated. The proportion of lost and damaged, MI, MII, and degenerate oocytes were compared between groups by Chi square test. Vitrification procedure increased ( $\mathrm{p}$ $\leq 0.05$ ) the percentage of oocytes lost and damaged when compared to control group. Additionally, vitrification increased ( $\mathrm{p} \leq 0.05)$ the proportion of MI and degenerated oocytes, and decreased the proportion of MII oocytes. Therefore, vitrification by the OPS method of partially matured bovine oocytes is a reliable alternative for the conservation of germinal cells from Chilean Red Friesian females.

KEY WORDS: Oocytes; Vitrification; in vitro maturation; Chilean Red Friesian cattle.

\section{REFERENCIAS BIBLIOGRÁFICAS}

Albarracín, J. L.; Morató, R.; Rojas, C. \& Mogas, T. Effects of vitrification in open pulled straws on the cytology of in vitro matured prepubertal and adult bovine oocytes. Theriogenology, 63(3):890-901, 2005.

Aman, R. R. \& Parks, J. E. Effects of cooling and rewarming on the meiotic spindle and chromosomes of in vitro-matured bovine oocytes. Biol. Reprod., 50(1):103-10, 1994.

de Souza, J. M. G.; Batista, R. I. T. P.; Melo, L. M. \& Freitas, V. J. F. Reproductive biotechnologies applied to the conservation of endangered ruminants - past, present and future. Rev. Port. Cienc. Vet., 106(577-580):31-8, 2011.

Dhali, A.; Manik, R. S.; Das, S. K.; Singla, S. K. \& Palta, P. Vitrification of buffalo (Bubalus bubalis) oocytes. Theriogenology, 53(6):1295-303, 2000.

Dinnyés, A.; Dai, Y.; Jiang, S. \& Yang, X. High developmental rates of vitrified bovine oocytes following parthenogenetic activation, in vitro fertilization, and somatic cell nuclear transfer. Biol. Reprod., 63(2):513-8, 2000.

Gordon, I. Laboratory Production of Cattle Embryos. $2^{\mathrm{a}}$ ed. Wallingford (Oxon), CABI Publishing, 2003.

Hamano, S.; Kokeida, A.; Kuwayama, M. \& Nagai, T. Full-term development of in vitro-matured, vitrified and fertilized bovine oocytes. Theriogenology, 38(6):1085-90, 1992.

Hochi, S.; Ito, K.; Hirabayashi, M.; Ueda, M.; Kimura, K. \& Hanada, A. Effect of nuclear stages during IVM on the survival of vitrified-warmed bovine oocytes. Theriogenology, 49(4):787-96, 1998.

Hou, Y. P.; Dai, Y. P.; Zhu, S. E.; Zhu, H. B., Wu, T. Y.; Gong, G. C.; Wang, H. P.; Wang, L. L.; Liu, Y.; Li, R.; Wan, R. \& Li, $\mathrm{N}$. Bovine oocytes vitrified by the open pulled straw method and used for somatic cell cloning supported development to term. Theriogenology, 64(6):1381-91, 2005.

Hyttel, P.; Vatja, G. \& Callesen, H. Vitrification of bovine oocytes with the open pulled straw method: ultrastructural consequences. Mol. Reprod. Dev., 56(1):80-8, 2000.

Li, X.; Su, L.; Li, Y.; Ji, W. \& Dinnyés, A. Vitrification of Yunnan Yellow Cattle oocytes: work in progress. Theriogenology, 58(7):1253-60, 2002.

Mapes, D. \& Wells, M. E. Maturation and Freezing of Bovine Oocytes. Anim. Sci. Res. Rep., 1977:161-5, 1977.

Martino, A.; Songsasen, N. \& Leibo, S. P. Development into blastocysts of bovine oocytes cryopreserved by ultra-rapid cooling. Biol. Reprod., 54(5):1059-69, 1996. 
Martins, R. D.; Costa, E. P.; Chagas, J. S. C.; Ignácio, F. S.; Torres, C. A. A. \& McManus, C. Effects of vitrification of inmature bovine oocytes on in vitro maturation. Anim. Reprod., 2(2):12834, 2005.

Men, H.; Monson, R. L. \& Rutledge, J. J. Effect of meiotic stages and maturation protocols on bovine oocyte's resistance to cryopreservation. Theriogenology, 57(3):1095-103, 2002.

Morató, R.; Izquierdo, D.; Paramio, M. T. \& Mogas, T. Embryo development and structural analysis of in vitro matured bovine oocytes vitrified in flexipet denuding pipettes. Theriogenology, 70(9):1536-43, 2008.

Otoi, T.; Yamamoto, K.; Koyama, N. \& Susuki, T. In vitro fertilization and development of immature and mature bovine oocytes cryopreserved by ethylene glycol with sucrose. Cryobiology, 32(5):455-60, 1995.

Prentice-Biensch, J. R.; Singh, J.; Mapletoft, R. J. \& Anzar, M. Vitrification of immature bovine cumulus-oocyte complexes: effects of cryoprotectants, the vitrification procedure and warming time on cleavage and embryo development. Reprod. Biol. Endocrinol., 10:73, 2012.

Rall, W. F. \& Fahy, G. M. Ice-free cryopreservation of mouse embryos at -196 degrees C by vitrification. Nature, 313(6003):573-5, 1985.

Saragusty, J. \& Arav, A. Current progress in oocyte and embryo cryopreservation by slow freezing and vitrification. Reproduction, 141(1):1-19, 2011.

Sharma, G. T. \& Loganathasamy, K. Effect of meiotic stages during in vitro maturation on the survival of vitrified-warmed buffalo oocytes. Vet. Res. Commun., 31(7):881-93, 2007.

Silva, M. E. \& Berland, M. A. Vitrificación de blastocitos bovinos producidos in vitro con el método Open Pulled Straw (OPS): Primer reporte. Arch. Med. Vet., 36:59-62, 2004.

Sirard, M. A.; Parrish, J. J.; Ware, C. B.; Leibfried-Rutledge, M. L. \& First, N. L. The culture of bovine oocytes to obtain developmentally competent embryos. Biol. Reprod., 39(3):546$52,1988$.

Smiljakovic, T. \& Tomek, W. Meiotic maturation and in vitro maturation of bovine oocytes. Biotech. Anim. Husb., 22(12):29-34, 2006.

Tharasanit, T.; Colleoni, S.; Lazzari, G.; Colenbrander, B.; Galli, C. \& Stout, T. A. Effect of cumulus morphology and maturation stage on the cryopreservability of equine oocytes. Reproduction, 132(5):759-69, 2006.

Vatja, G.; Holm, P.; Greve, T. \& Callesen, H. Vitrification of porcine embryos using the Open Pulled Straw (OPS) method. Acta Vet. Scand., 38(4):349-52, 1997a.
Vatja, G.; Booth, P. J.; Holm, P.; Kuwayama, M.; Greve, T. \& Callesen, H. Succesfull vitrification of early stage bovine in vitro produced embryos with the Open Pulled Straw (OPS) method. Cryoletter, 18:191-5, 1997b.

Vatja, G.; Holm, P.; Kuwayama, M.; Booth, P. J.; Jacobsen, H.; Greve, T. \& Callesen, H. Open Pulled Straw (OPS) vitrification: a new way to reduce cryoinjuries of bovine ova and embryos. Mol. Reprod. Dev., 51(1):53-8, 1998.

Vieira, A. D.; Mezzalira, A.; Barbieri, D. P.; Lehmkhul, R. C.; Rubin, M. I. \& Vatja, G. Calves born after open pulled straw vitrification of immature bovine oocytes. Cryobiology, 45(1):91-4, 2002.

Zhou, X. L.; Al Naib, A.; Sun, D. W. \& Lonergan, P. Bovine oocyte vitrification using the Cryotop method: effect of cumulus cells and vitrification protocol on survival and subsequent development. Cryobiology, 61(1):66-72, 2010.

\section{Dirección para Correspondecia: Mauricio Silva \\ Escuela de Medicina Veterinaria Facultad de Recursos Naturales Universidad Católica de Temuco Casilla 15-D \\ Temuco \\ CHILE}

Email: masilva@uct.cl

Recibido : 04-01-2016

Aceptado: 04-05-2016 